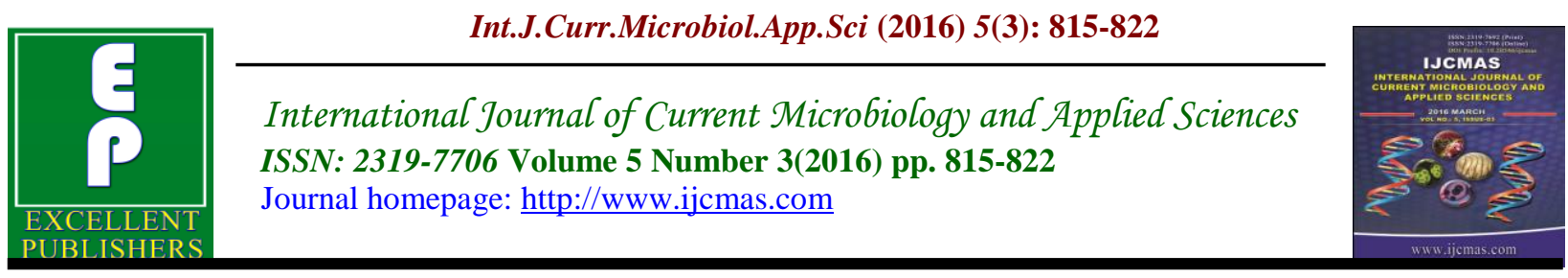

Original Research Article

http://dx.doi.org/10.20546/ijcmas.2016.503.094

\title{
Community Acquired Pneumonia is not free from Complications-A Tertiary Care Hospital Scenario
}

\author{
A.V. Sowmya ${ }^{1 *}$, G. Jayalakshmi ${ }^{2}$ and David Agatha ${ }^{2}$ \\ ${ }^{1}$ Department of Microbiology, Government Chengalpatttu Medical College, \\ Chengalpattu, Tamilnadu, India \\ ${ }^{2}$ Institute of Microbiology, Madras Medical College, Chennai, Tamilnadu, India \\ *Corresponding author
}

\begin{abstract}
A B S T R A C T
Keywords

Community

acquired

pneumonia,

Complications,

Multidrug

resistance.

\section{Article Info}

Accepted:

22 February 2016

Available Online:

10 March 2016

Pneumonia is one of the most common public health infectious disease and one of the major cause of morbidity and mortality in elderly and immunocompromised(IC) patients. The mortality rate increases considerably when pneumonia is associated with the development of complications. This study is conducted to identify the current patterns of Community Acquired Pneumonia (CAP) complications, associated IC states and to analyze its microbiological profile. Patients above 18 years presenting with signs and symptoms of pneumonia during the study period were included. Associated complication patterns were documented. Various respiratory samples were collected aseptically and processed according to Standard Operating Procedures. The isolated microbial agents and their antimicrobial susceptibility pattern were identified according to CLSI guidelines. Out of the total 150 patients in the study group, $19.99 \%$ of them presented with one of the complications of CAP. Diabetes mellitus (48\%) was the most common associated IC state followed by hematological malignancies $(18.66 \%)$. Most common complication is bacteremia (9.33\%), followed by pleural effusion (6\%). Complications are more common in patients with associated IC state (70\%) than in patients who are immunocompetant (IP) (30\%) with statistical significance. Multidrug Resistant organisms are more commonly associated with complications (73.33\%). Methicillin Resistant Staphylococcus aureus (36.67\%) and Klebsiella pneumoniae (30\%) were the most common bacterial agents identified in association with pneumonia complications. With antibiotic therapy being the main stay of treatment in CAP and with definitive treatment patterns for associated complications, proper knowledge of host factors, currently prevailing complication patterns, etiological agents and their resistance pattern pave an effective way to formulate empirical antibiotic therapy.
\end{abstract}

\section{Introduction}

One of the most challenging aspects in clinical medicine is Pneumonia which is characterized as consolidation pathologically and clinically as constellation of signs and symptoms. Pneumonia was described 2,500 years ago by Hippocrates 
and Dr.William Osler, described pneumonia as the "captain of the men of death".Community Acquired Pneumonia (CAP) is a major and significant cause of mortality and morbidity,in a developing country like India. Over 155 million cases of pneumonia and 1.8 million deaths occur annually worldwide (WHO, 2009). In India, there are no recent large studies conducted to determine the incidence and prevalence of CAP. Pneumonia in adults is estimated to be prevalent in about $4 \%$ of Indian population with male to female ratio of $1.56: 1.14$ (WHO, South East Asia region,2012)and an incremental annual incidence rate was observed (1.12-3.16 per 1000 inhabitants), the incidence being increased with age (A lmirall J et al., 2000). However the mortality rates of CAP in India varies between 3.3\%$11 \%$, as evidenced by several studies. Early diagnosis and rapid implementation of antimicrobial therapy plays a key role in treatment and also in improving the prognosis.CAP commonly present with complications like pleural effusion, lung abscess, empyema, bacteremia, especially whenever it is associated with late presentation, presence of risk factors and wrong choice of empirical antibiotics. These complications may be life threatening and may need proper directed therapy. Mortality rate is significantly higher in CAP patients presenting with complications (24\%) than CAP patients without complications $(11 \%)$ (GC Mbata et al., 2013).Our study aimed to identify the current common complication patterns of CAP, associated immunocompromised (IC) state and to analyze its microbiological profile in adults patients, in a tertiary care hospital.

\section{Materials and Methods}

The study was conducted in a tertiary care hospital over a period of one year, after getting ethical clearance. Patients above 18 yrs who were admitted in various wards of the hospital with presenting signs and symptoms of high grade fever, chills and rigors, cough with sputum production, dyspnea, tachycardia, tachypnea, focal signs in physical examination and chest radiography infiltrates were included in the study group. After getting informed consent, demographic details along with associated IC state if any, were documented by structured questionnaire. Associated complication patterns and basic blood investigation details like Hemoglobin estimation, Total leukocyte count, Differential count, peripheral smear examination report, Blood glucose level, Blood urea and Serum creatinine levels were documented.

Various respiratory samples like sputum/induced sputum, Broncho Alveolar Lavage fluid, bronchial wash, cavity material and pleural fluid were collected under strict aseptic precautions and were sent to the microbiology department for culture and sensitivity testing. Blood samples were collected from all the patients for culture and sensitivity testing. The samples were inoculated into various culture media like MacConkey agar, Blood agar and Chocolate agar and the isolated organisms were identified by standard procedures. Antimicrobial sensitivity testing was done according to CLSI guidelines and resistance patterns were identified. The results were analyzed statistically.

\section{Results and Discussion}

150 patients who presented with signs \& symptoms suggestive of pneumonia during one year period and were included in the study group. About $50 \%$ of the patients have associated IC state and $50 \%$ of the study group had no associated IC state and were considered immunocompetant (IP). Majority 
of the patients were males $(66.66 \%)$ and most of the study population were in the age group of 21-30yrs (22.66\%) followed by 51$60 y r s(18.67 \%)$.

Associated immunocompromised (IC) states were identified in 50\% of our study population $(n=75)$. Diabetes Mellitus was the most common associated IC state (48\%), followed by patients with hematological malignancies (18.66\%). Other IC states noted in this group are autoimmune diseases $(10.66 \%)$, post renal transplant patients (10.66\%), patients on prolonged steroid therapy $(5.33 \%)$, retroviral disease positive patients (4\%) and patients with solid organ malignancy $(2.66 \%)$.

Etiological agents was identified in 104 cases $(69.33 \%)$ with respiratory sample analysis and 14 cases gave positive results in blood culture $(9.33 \%)$. The etiologic agent identified in the respiratory sample and the corresponding blood sample were one and the same with same antimicrobial sensitivity pattern. The most commonly isolated etiologic agent was Klebsiella pneumoniae (36, 34.62\%) followed by Staphylococcus aureus $(15,14.42 \%)$.

Of 150 cases of CAP, 30 cases (19.99\%) presented with complications, the most common being bacteremia (9.33\%), followed by pleural effusion (6\%) (Table 1). Complications are common in patients with associated IC state $(70 \%)$ than in patients without associated IC state i.e. in IP patients $(30 \%)$ with significant $\mathrm{P}$ value $(\mathrm{P}=0.035)$. Bacteremia is the most common complication in IP (44.44\%) and in IC $(47.62 \%)$ patients with no statistically significant difference in the prevalence of bacteremia, between the IP and IC patients $(\mathrm{P}=0.215)$ (Table 2)

Klebsiella pneumoniae (ESBL) is the most common pathogen isolated from blood in IP patients (50\%).Staphylococcus aureus (MRSA) is the most common pathogen isolated from blood in IC patients (50\%).Multidrug resistant organisms are more commonly associated with the development of complications $(22,73.33 \%)$ (Table 2) $70 \%$ of bacteremia, $50 \%$ of pleural effusion, $100 \%$ of lung abscess and $75 \%$ of empyema complications are seen in association with diabetes mellitus (Chart1).

Pneumonia is an infection of the lung parenchyma caused by proliferation of microbial pathogens at the alveolar level and the clinical manifestations are due to varied host responses towards these proliferating pathogens. Worldwide $>600,000$ individuals hospitalize every year due to pneumonia with annual deaths of 45, 000, the rate increases with presence of associated IC state and complications (Rano et al., 2005). Males are more frequently affected due to the presence of associated risk factors like smoking, COPD etc...Majority of the people in the study population were males, with most of them were in the age group of 21-30 yrs. People having associated IC state fall in the age group of 51-60yrs, due to the presence of chronic diseases, predisposing them to infections. Diabetes mellitus (DM) is the most common associated IC state (48\%) with CAP followed by hematological malignancies (18.66\%).Study conducted by Reimar Wernich Thomsen (2004) for his $\mathrm{PhD}$ thesis on $\mathrm{DM}$ and Community acquired bacteremia, showed that DM is the most common IC state associated with CAP and has1.32 fold higher risk for contracting pneumonia than control groups due to the associated abnormalities in immune functions.

$19.99 \%$ of the study population presented with one of the complication of CAP (Table 1). Patients with associated IC state had presented with more number of complications $(70 \%)$ than IP patients $(30 \%)$ 
with statistical significance (Table 2). Many such studies, like the ones conducted by American Lung Association group (2008),on understanding pneumonia and Ann Pietrangelo (2012) paper on pneumonia in immunocompromised host, Health line references, explained that patients with weakened immune system have a 2 fold increased risk in developing complications of pneumonia with significant morbidity and mortality.

Bacteremia was the most common complication $(9.33 \%)$, followed by pleural effusion (6\%) (Table 1). Our data does not correlate with the study conducted by GC Mbata et al. (2013), who showed that complications were observed in $31.3 \%$ of CAP patients, with pleural effusion being the commonest complication (25\%) followed by bacteremia (6.25\%). Also studies conducted by Nwosu CM et al., (1991) and Jennings LC et al. (2008) agrees that pleural effusion is the most common complication. However there is no statistical significance in the occurrence of bacteremia between IC group (47.62\%) and IP group $(44.44 \%)$ in our study as evidenced by study conducted by D.Sousa et al. (2012). ESBL producing Klebsiella pneumoniae is the most common pathogen causing bacteremia in IP group $(50 \%)$ whereas Methicillin resistant Staphylococcus aureus is the most common pathogen causing bacteremia in IC group $(50 \%)$ (Table 2$) .10 \%$ of bacteremia in IC group is caused by S.pneumoniae, in the current study. Study conducted by Reimar Wernich (2004) on his $\mathrm{PhD}$ thesis, shows that bacteremia is commonly caused by gram negative organisms in patients with weakened immune system, which is not correlating with our study data. However study conducted by Spomenka Ljubic et al. (2004) and Byoko EJ et al. (1989), found out that patients with weakened immune system are associated with gram positive bacteremia, particularly with Staphylococcus aureus and Streptococcus pneumoniae which correlates well with our data.

In our current study, Pleural effusion contributes for $6 \%$ of CAP complication(Table 1).Gram positive organisms are identified as causative agents in $55.56 \%$,with Gram negatives comprising about $44.44 \%$.Our finding correlates well with study conducted by Tsang KY et al., (2007), which shows that Staphylococcus aureus and Streptococcus pneumonaie comprise about $70 \%$ of parapneumonic effusions. The significant detection of Gram negatives as etiologic agents of pleural effusion in our current study indicates that this changing pattern of microbial agents has to be borne in mind while formulating empirical antibiotic therapy in people presenting with pneumonia complications.

Empyema compromise about $3.33 \%$ of complications in our study with Gram positives accounting for $60 \%$ of etiologic agents (Table 1,2). Staphylococcus aureus was the most common isolated pathogen $(40 \%)$. As indicated in various studies, bacterial agents accounts for about $70 \%$ of empyema cases, with S.aueus \& S.pneumoniae as the commonest agents. They have also documented that Gram negatives like Pseudomonas, Klebsiella and other enterobacteriaceae organisms are also being encountered as common agents causing empyema (Chapman SJ et al., 2004; Sahn SA, 2007; Bartlett JG et al., 1974).

Lung abscess accounts for about $1.33 \%$ of complications in our study with S.aureus (MRSA)and Klebsiella pneumoniae (ESBL) accounting for $50 \%$ of etiologic agent each (Table 1,2). Till now anaerobic agents and polymicrobial infection are the common agents of lung abscess. As evidenced by various studies, among aerobic etiologic 
agents of lung abscess MRSA, Streptococcus pyogenes, Streptococcus pneumoniae, Klebsiella pneumoniae, Pseudomonas aeruginosa and H.influenzae are the commonest agents, a fact which correlates well with our study (Wang JL et al., 2005; Ivan Kuhajda et al., 2015).

Multidrug resistant organisms (MDR) are more commonly isolated in our patients presenting with complications (73.33\%) (Table 2). Methicillin Resistant
Staphylococcus aureus (MRSA) (36.67\%) and Klebsiella pneumoniae (ESBL) (30\%) were the most common bacterial agents identified in our study, in association with pneumonia complications. About $77.77 \%$ of organisms involved in complications of CAP in IP patients are MDR organisms when compared to $71.43 \%$ in IC patients, without any statistical significance. Study conducted by Saibal MAA et al. (2012) showed that diabetic patients are more prone to develop CAP due to MDR organisms.

Table.1 Distribution of Complications of Pneumonia among the Study Population $(n=150)$

\begin{tabular}{|c|c|c|c|}
\hline Sl.no & Complications & No & \% \\
\hline 1. & Bacteremia & 14 & 9.33 \\
\hline 2. & Pleural effusion & 9 & 6 \\
\hline 3. & Empyema & 5 & 3.33 \\
\hline 4. & Lung abscess & 2 & 1.33 \\
\hline & Total & 30 & 19.99 \\
\hline
\end{tabular}

Table.2 Correlation of Complications with Etiologic Agents

\begin{tabular}{|c|c|c|c|c|c|c|c|c|}
\hline $\begin{array}{l}\text { Study } \\
\text { group }\end{array}$ & Complication & MSSA & MRSA & $\begin{array}{l}\text { S. } \\
\text { pneumoniae }\end{array}$ & $\begin{array}{l}\text { S. } \\
\text { viridans }\end{array}$ & $\begin{array}{l}\text { K. } \\
\text { pneumoniae } \\
\text { (ESBL) }\end{array}$ & $\begin{array}{l}\text { K. } \\
\text { pneumoniae } \\
\text { (AmpC) }\end{array}$ & $\begin{array}{l}P . \\
\text { aeruginosa }\end{array}$ \\
\hline \multirow{4}{*}{$\begin{array}{l}\text { Without } \\
\text { associated } \\
\text { IC state-IP } \\
(\mathbf{9}, \mathbf{3 0 \%})\end{array}$} & $\begin{array}{l}\text { Bacteremia } \\
(n=4)\end{array}$ & - & $\begin{array}{l}1 \\
(25 \%)\end{array}$ & & $1(25 \%)$ & $2(50 \%)$ & & \\
\hline & $\begin{array}{l}\text { Pleural } \\
\text { effusion }(n=3)\end{array}$ & & $\begin{array}{l}1 \\
(33.33 \%)\end{array}$ & $1(33.3 \%)$ & & $1(33.33 \%)$ & & \\
\hline & $\begin{array}{l}\text { Empyema } \\
(n=1)\end{array}$ & & & & & $1(100 \%)$ & & \\
\hline & $\begin{array}{l}\text { Lung abscess } \\
(n=1)\end{array}$ & & $1(100 \%)$ & & & & & \\
\hline \multirow{5}{*}{$\begin{array}{l}\text { With } \\
\text { associated } \\
\text { IC state } \\
(\mathbf{2 1 , 7 0 \% )}\end{array}$} & $\begin{array}{l}\text { Bacteremia } \\
(\mathbf{n}=\mathbf{1 0})\end{array}$ & $\begin{array}{l}1 \\
(10 \%)\end{array}$ & $5(50 \%)$ & $1(10 \%)$ & & $2(20 \%)$ & $1(10 \%)$ & \\
\hline & $\begin{array}{l}\text { Pleural } \\
\text { effusion }(n=6)\end{array}$ & & $\begin{array}{l}1 \\
(16.66 \%)\end{array}$ & $2(33.33 \%)$ & & $2(33.33 \%)$ & $1(16.66 \%)$ & \\
\hline & $\begin{array}{l}\text { Empyema } \\
(n=4)\end{array}$ & & $2(50 \%)$ & $1(25 \%)$ & & & & $1(25 \%)$ \\
\hline & $\begin{array}{l}\text { Lung abscess } \\
(n=1)\end{array}$ & & & & & $1(100 \%)$ & & \\
\hline & Total & 1 & 11 & 5 & 1 & 9 & 2 & 1 \\
\hline
\end{tabular}

MSSA-Methicillin Sensitive Staphylococcus aureus.MRSA-Methicillin Resistant Staphylococcus aureus 
Chart.1 Association of Complications of CAP with Immunocompromised States

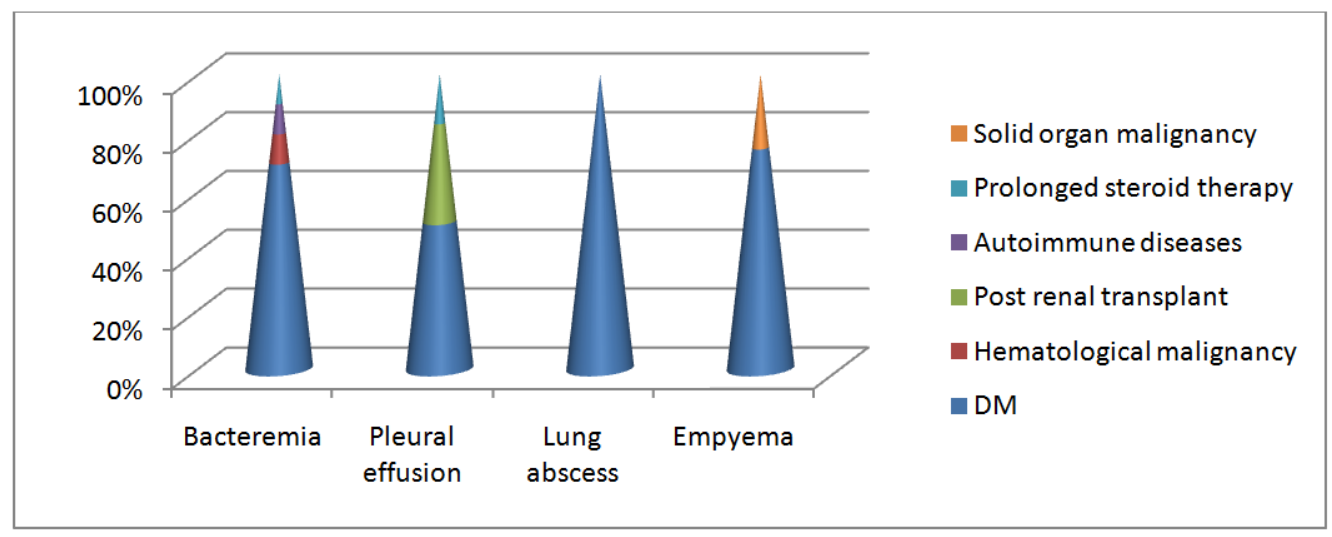

This might be due to the fact of improper empirical therapy, without switching over to specific treatment following culture \& sensitivity report paving a background for selective proliferation of MDR organisms. Improper empirical therapy are not effective in patients with MDR etiology and this may lead to occurrence of more complications in pneumonia

In the present study about correlation of complications with immunocompromised state, $70 \%$ of bacteremia, $50 \%$ of pleural effusion and $75 \%$ of empyema complications are found in association with diabetic patients (Chart 1).This finding is supported by studies conducted on pulmonary infections in diabetes mellitus by Spomenka Ljubic et al., (2004) and Koziel $\mathrm{H}$ et al. (1995) which showed that pulmonary complications are more frequent in diabetic individuals, about 10 times higher when compared to non diabetic individuals. Diabetes mellitus seems to be the single most important risk factor for developing complications of pneumonia.

In conclusion, Pneumonia is one of the leading cause of morbidity throughout world and especially so in developing countries, with different epidemiology, pathogenesis and clinical course. As the key to proper treatment in CAP is antibiotics, identification of etiologic agent with their antimicrobial susceptibility pattern is of utmost importance. Often based on the host factors, clinical setting and current patterns of involved pathogens with their local susceptibility patterns, empirical antibiotics are started. Treatment should be initiated as early as possible with specific antibiotics as CAP is frequently associated with complications.

Complications in CAP depend on the infecting pathogen and patient health with its own attendant risk of morbidity and mortality. IC patients have more chance of developing CAP complications, especially with MDR organisms and a higher mortality rate than with IP patients. Hence knowledge of the common current complication patterns of CAP, host factors, changing patterns of etiologic agents along with their resistant pattern allows the clinician to properly aim their empirical therapy in patient care and also in prevention of these complications in predisposed patients.

India being the country housing largest number of diabetic and other IC patients, a prior knowledge about the complication 
patterns of CAP and pathogens will lead to prevention and proper management of complications of CAP and hence to reduce the mortality rates. Also it is quiet clear that various life style modifications are needed in the current scenario and this will pave way in reducing the alarming emergence of these immunocompromised states.

Emergence of MDR bacteria that cause pneumonia in the community has created the need to identify risk factors for acquiring resistant pathogens and to identify those patients who need an empirical broadspectrum antibiotic therapy. This in turn will help the clinicians to culminate the associated complications and hence mortality rates in community-onset pneumonia.

\section{References}

Almirall, J., Bolíbar, I., Vidal, J., et al. 2000. Epidemiology of community acquired pneumonia in adults: A population-based study. Eur. Respir. J., 15: 757-763.

Bartlett, J.G., Gorbach, S.L., Thadepalli, H., Finegold, S.M. 1974. Bacteriology of empyema. Lancet, 1(7853): 33840.

Boyko, E.J., Lipsky, B.A., Sandoval, R. et al. 1989. NIDDM and prevalence of nasal S.aureus colonisation. Diabetes care, 12: 189-193.

Chapman, S.J., Davies, R.J. 2004. Recent advances in parapneumonic effusion and empyema. Curr. Opin. Resp. Med., 10: 299-304.

Department of Communicable Diseases, Regional Office for South East Asia Region, World Health Organisation, New Delhi, India. 2012. Pneumonia in South-East Asia Region: Public health perspective. Indian $\mathrm{J}$. Med. Res., 135: 459-468.
Sousa, D., Justo, I., Domínguez, A., Manzur, A. 2012. Community-acquired pneumonia in immunocompromised older patients: incidence, causative organisms and outcome. Clin. Microbiol. Infec., doi: 10.1111/j.1469-0691.2012.03765.

Mbata, G.C., Chukwuka, C.J., Onyedum, C.C., Onwubere, B.J.C. 2013. The Role of Complications of Community Acquired Pneumonia on the Outcome of the Illness: A Prospective Observational Study in a Tertiary Institution in Eastern Nigeria. Ann. Med. Health Sci. Res., 3(3): 365-369.

Ivan Kuhajda, Konstantinos Zarogoulidis, Katerina Tsirgogianni, Drosos Tsavlis, Ioannis Kioumis, Christoforos Kosmidis, et al. 2015. Lung abscess-etiology, diagnostic and treatment options. Ann. Transl. Med., 3(13): 183. doi: 10.3978/j.issn.2305-5839. 2015.07.08.

Jennings, L.C., Anderson, T.P., Beynon, K.A., Chua, A., Laing, R.T., Werno, A.M., et al. 2008. Incidence and characteristics of viral communityacquired pneumonia in adults. Thorax, 63: 42-8.

Koziel, H., Koziel, M.J. 1995. Pulmonary complications of diabetes mellitus. Infect. Dis. Clin. North Am., 9(1): 65-96.

Nwosu, C.M., Anisuba, B.C. 1991. A hospital study of adults with CAP 11-clinical course and complications. Orient J. Med., 3: 196-9.

Rano, A., Agusti, C., Sibila, O., Torres, A. 2005. Pulmonary infections in nonHIVimmunocompromised patients. Curr. Opin. Pulm. Med., 11(3): 2137.

Sahn, S.A. 2007. Diagnosis and management of parapneumonic 
effusions and empyema. Clin. Infect. Dis., 45(11): 1480-6.

Saibal, M.A.A., Rahman, S.H.Z., Nishat, L., Sikder, N.H., Begum, S.A., Islam, M.J. et al. 2012. Community acquired pneumonia in diabetic and non-diabetic hospitalized patients: presentation, causative pathogens and outcome. Bangladesh Med. Res. Counc. Bull., 38: 98-10.

Spomenka Ljubic, Ayyasamy Balachandran. 2004. Pulmonary infections in Diabetes Mellitus. Diabetologia Croatica, 33(4): 115-124.

Tsang, K.Y., Leung, W.S., Chan, V.L., Lin, A.W., Chu, C.M. 2007. Complicated parapneumonic effusion and empyema thoracis: microbiology and predictors of adverse outcomes. Hong Kong Med. J., 13(3): 178-86.

Wang, J.L., Chen, K.Y., Fang, C.T., et al. 2005. Changing bacteriology of adult community-acquired lung abscess in Taiwan: Klebsiella pneumoniae versus anaerobes. Clin. Infect. Dis., 40: 915-22.

World Health Organization. 2009. Fact sheet number 331 - Pneumonia. Available at: http://www.who.int/mediacentre/ factsheets/fs331/en/index.html.

\section{How to cite this article:}

Sowmya, A.V., Jayalakshmi, G. and David Agatha. 2016. Community Acquired Pneumonia is not Free from Complications-A Tertiary Care Hospital Scenario. Int.J.Curr.Microbiol.App.Sci. 5(3): 815-822. doi: http://dx.doi.org/10.20546/ijcmas.2016.503.094 